\section{Multidimensional sorting and matrix display}

\author{
D. LOUIS WOOD \\ University of Arkansas, Little Rock, Arkansas
}

McNally and Wood (1982) introduced a short, fast-running program that is written in Level II BASIC for the TRS-80 Model III and that ranks scores in a single group and resolves any number of ties. The present program, illustrated in Table 1 , generalizes that technique to accommodate an $\mathrm{M} \times \mathrm{N}$ matrix and uses Kendall's W to illustrate the process. It was written in Level II BASIC for the TRS-80 Model 4.

When it is run, the program asks for the number of subjects, that is, scores, per column (N) and the number of judges (M). These values are used to initialize an $\mathrm{M} \times \mathbf{N} \times 3$ array, for which the maximum values of $M$ and $N$ are determined by the available RAM. The program then asks that the scores be entered and stores them in the first line of the third dimension of the array. Line 10 determines ranks, and lines 13 and 15 resolve any number of ties. Ranks are stored in the second line of the third dimension, and the frequency of occurrence is resident in the third line. Lines 17-19 cause the $\mathrm{M} \times \mathrm{N}$ rank matrix to be printed; they contain an interesting feature. The TRS-80 Model 4 responds to multiple-loop print statements of the type in lines $17-19$ by rearranging the matrix on the screen in a confusing manner. The PRINT " " statement in line 19 suppresses the rearrangement and correctly prints the rank matrix for $2<M \leqslant 15$. In cases in which the number of judges exceeds 15 , the display of the rank matrix is scrambled, but the ranks, the value of $W$, and the summary table are unaffected.

Lines 20-30 compute $W$ and the contents of the summary table, which displays the sum of all the judges' ranks for each $S$, the difference of each rank sum (D) from the mean (AVE) of the rank sums, and the $D$ squared column. The sum of the D squares is listed for use in consulting a significance table.

The author's mailing address is: Department of Psychology, University of Arkansas, Little Rock, Arkansas.
Table 1

Multidimensional Sorting and Matrix-Display Program

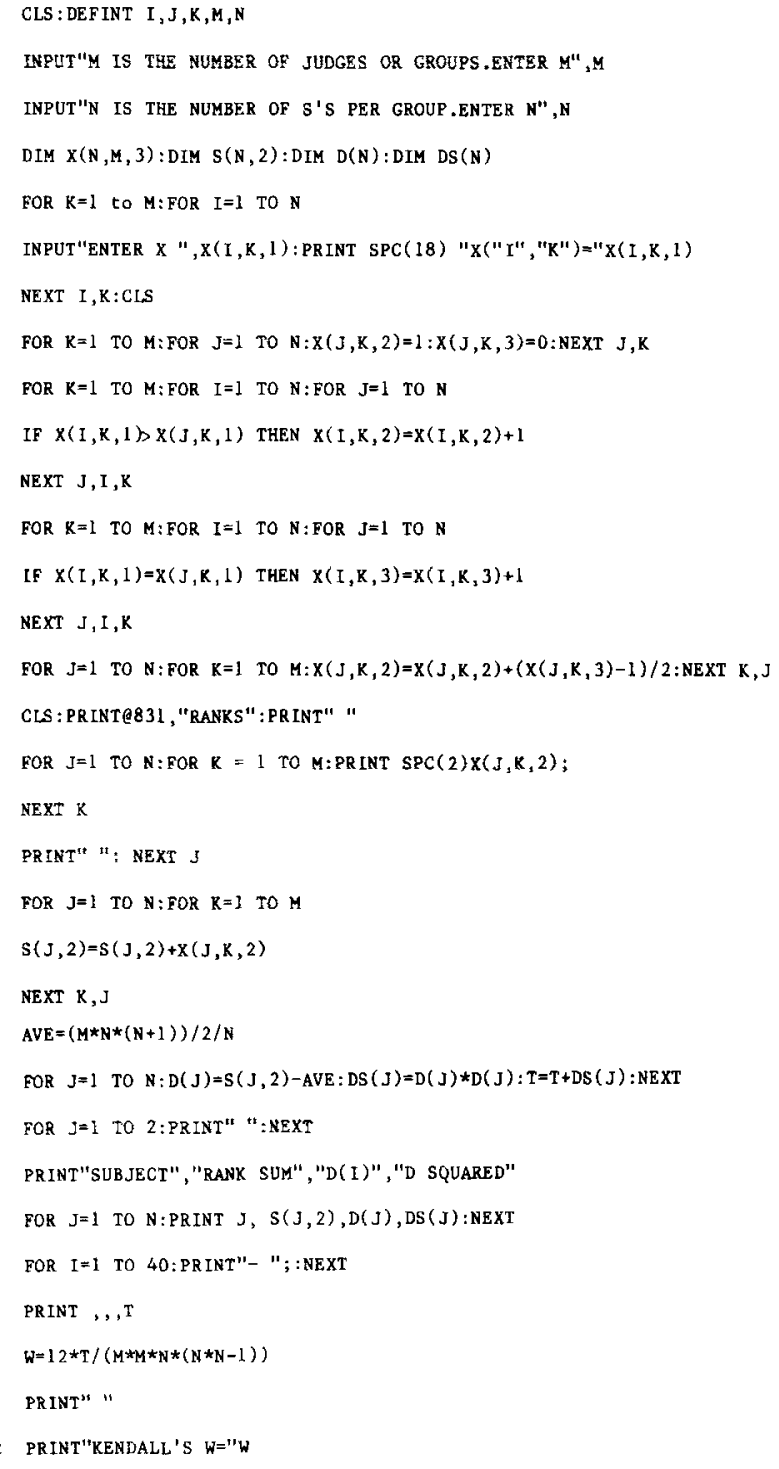

\section{REFERENCE}

McNally, K. A., \& Wood, D. L. BASIC rank-order program. Behavior Research Methods \& Instrumentation, 1982, 14, 545.

(Manuscript accepted for publication August 4, 1983.) 